\title{
Orbital period decay of compact black hole X-ray binaries: the influence of circumbinary disks? (Research Note)
}

\author{
Wen-Cong Chen ${ }^{1,3}$ and Xiang-Dong $\mathrm{Li}^{2,3}$ \\ ${ }^{1}$ School of Physics and Electrical Information, Shangqiu Normal University, 476000 Shangqiu, PR China \\ e-mail: chenwc@nju.edu.cn \\ 2 Department of Astronomy, Nanjing University, 210093 Nanjing, PR China \\ 3 Key Laboratory of Modern Astronomy and Astrophysics (Nanjing University), Ministry of Education, 210093 Nanjing, PR China \\ Received 13 May 2015 / Accepted 19 August 2015
}

\section{ABSTRACT}

\begin{abstract}
Context. Recently, compact black hole X-ray binaries XTE J 1118+480 and A0620-00 have been reported to be experiencing a fast orbital period decay, which is two orders of magnitude higher than expected with gravitational wave radiation. Magnetic braking of an Ap/Bp star has been suggested to account for the period change when the surface magnetic field of the companion star $B_{\mathrm{s}} \gtrsim 10^{4} \mathrm{G}$. However, our calculation indicates that anomalous magnetic braking cannot significantly contribute to the large orbital period decay rates observed in these two sources even if $B_{\mathrm{s}} \gtrsim 10^{4} \mathrm{G}$.

Aims. Observations have provided evidence that circumbinary disks around two compact black hole X-ray binaries may exist. Our analysis shows that, for some reasonable parameters, tidal torque between the circumbinary disk and the binary can efficiently extract the orbital angular momentum from the binary, and result in a large orbital period change rate.

Methods. Based on the circumbinary disk model, we simulate the evolution of XTE J 1118+480 via a stellar evolution code.

Results. Our computations are approximatively in agreement with the observed data (the masses of two components, donor star radius, orbital period, and orbital period derivative).

Conclusions. The mass transfer rate and circumbinary disk mass are obviously far greater than the inferred values from observations. Therefore, it seems that the circumbinary disk is unlikely to be the main cause of the rapid orbital decay observed in some compact black hole X-ray binaries.
\end{abstract}

Key words. stars: black holes - stars: evolution - stars: mass-loss - X-rays: binaries

\section{Introduction}

There are 18 stellar-mass black hole X-ray binaries (BHXBs) in our Galaxy (Remillard \& McClintock 2006; Casares 2007). Half of these have short orbital periods $(\lessgtr 0.5 \mathrm{~d})$ and light donor stars (of mass $\lesssim 1.0 M_{\odot}$ ), and are classified as compact BHXBs (Lee et al. 2002; Podsiadlowski et al. 2003; Ritter \& Kolb 2003). At present, the origin of compact BHXBs is still controversial, although it is accepted that these binaries must have experienced common envelope evolution to account for current short orbital periods. According the theory, low-mass stars, however, are not able to eject the envelope of the black hole progenitors during the common envelope evolution stage (Podsiadlowski et al. 2003). Therefore, it was suggested that compact BHXBs may have evolved from BHXBs with an intermediate-mass $\left(\gtrsim 2-3 M_{\odot}\right)$ donor star, i.e., black hole intermediate-mass X-ray binaries (BHIMXBs). This viewpoint was supported by the observation of CNO-processed material in XTE J1118+480 (Haswell et al. 2002).

Generally, an intermediate-mass star with a radiative envelope cannot develop large-scale magnetic fields and undergo magnetic braking (Kawaler 1988). During the evolution of BHIMXBs, the orbital period should increase because material is transferred from the less massive secondary to the more massive black hole. Short orbital periods imply that efficient angular momentum loss mechanism must take place during the evolution of BHIMXBs.
As an alternative scenario, BHIMXBs with Ap/Bp stars, in which the donor stars anomalously have strong magnetic fields of $10^{2}-10^{4} \mathrm{G}$, could experience magnetic braking by the coupling between the magnetic field and the winds from the donor star driven by X-ray irradiation from the black holes (Justham et al. 2006). This can cause secular decrease in the orbital periods through tidal torques, although the efficiency of tidal interaction between the component with a radiative envelope and the orbit is uncertain (Dervişoğlu et al. 2010). Subsequently, Chen \& Li (2006) alternatively proposed that a circumbinary disk around the BHXBs may offer an efficient mechanism to extract angular momentum from the binary, driving BHIMXBs into compact BHXBs.

Recently, González Hernández et al. (2012) showed that the compact BHXB XTE J1118+480 is undergoing rapid orbital decay at a rate $\dot{P}=-1.83 \pm 0.66 \mathrm{~ms} \mathrm{yr}^{-1}$. Recently, the orbital period derivative was updated to be $\dot{P}=-1.90 \pm 0.57 \mathrm{~ms} \mathrm{yr}^{-1}$ (González Hernández et al. 2014). It was found that the orbit of A0620-00 is also shrinking, though at a slightly smaller rate, i.e, $\dot{P}=-0.60 \pm 0.08 \mathrm{~ms} \mathrm{yr}^{-1}$ (González Hernández et al. 2014). In Table 1, we list the physical parameters of these two compact BHXBs.

In this work, we attempt to investigate whether the circumbinary disk can drive the rapid decay of the orbital period measured in these binaries. In Sect. 2 we estimate the orbital period derivatives in the anomalous magnetic braking and circumbinary 
disk models, and show that the former is not likely to work for reasonable wind loss rates. In Sect. 3, we simulate the evolutionary sequences of XTE J1118+480, based on the circumbinary disk model. Section 4 contains a brief summary and discussion.

\section{The orbital decay and possible angular momentum loss mechanisms}

We consider that a BHXB consists of a BH of mass $M_{\mathrm{bh}}$ and a donor star of mass $M_{\mathrm{d}}$ in a circular orbit. If the mass transfer is conserved, the orbital period change is governed by the following equation:

$\frac{\dot{P}}{P}=3 \frac{\dot{J}}{J}-3 \frac{\dot{M}_{\mathrm{d}}}{M_{\mathrm{d}}}\left(1-\frac{M_{\mathrm{d}}}{M_{\mathrm{bh}}}\right)$,

where $J=2 \pi a^{2} M_{\mathrm{bh}} M_{\mathrm{d}} /\left(M_{\mathrm{T}} P\right)$ is the total orbital angular momentum of the binary, $a=\left(G M_{\mathrm{T}} P^{2} / 4 \pi^{2}\right)^{1 / 3}$ is the binary separation, $G$ is the gravitational constant, and $M_{\mathrm{T}}$ is the total mass of the binary. Since $\dot{M}_{\mathrm{d}}<0$, and $M_{\mathrm{d}} \ll M_{\mathrm{bh}}$, the second term on the right side of Eq. (1) always causes the orbital period to increase at a rate $\dot{P} \approx-3 P \dot{M}_{\mathrm{d}} / M_{\mathrm{d}}$. For a mass transfer rate $\sim 10^{-9} M_{\odot} \mathrm{yr}^{-1}$, this would indicate a positive period derivative of $\sim 0.24 \mathrm{~ms} \mathrm{yr}^{-1}$ and $\sim 0.20 \mathrm{~ms} \mathrm{yr}^{-1}$ for XTE J1118+480 and A0620-00, respectively. Therefore, the observed rapid orbital decay implies that an efficient angular momentum loss mechanism exists during the evolution BHXBs.

\subsection{Magnetic braking}

Magnetic braking originated from the coupling between the stellar winds and the magnetic field of the donor star (Verbunt \& Zwaan 1981). It was proposed that the orbital decay of XTE J1118+480 may be accounted for by magnetic braking if the donor star possesses an anomalously strong magnetic field of $B_{\mathrm{s}} \geq 10-20 \mathrm{kG}$, and the irradiation-driven wind loss rate from the donor star is comparable to the mass transfer rate (González Hernández et al. 2012). According to Eq. (15) in Justham et al. (2006), we can obtain the orbital period derivative caused by magnetic braking as follows:

$\dot{P}_{\mathrm{MB}}=-\frac{3}{2 \pi G^{1 / 4}} B_{\mathrm{s}} P^{2} \psi^{1 / 2} \frac{\dot{M}_{\mathrm{d}}^{1 / 2} M_{\mathrm{T}}^{3 / 2} R_{\mathrm{d}}^{15 / 4}}{M_{\mathrm{bh}} M_{\mathrm{d}}^{7 / 4} a^{7 / 2}}$,

where $R_{\mathrm{d}}$ is the radius of the Ap/Bp stars, $\psi \simeq 10^{-6} c^{2}$ is a synthetic parameter taking into account the fraction of the X-ray flux that intercepts the donor, the wind driving energy efficiency, and the rest mass to energy conversion efficiency.

In Fig. 1, we plot the calculated rate of orbital period change of XTE J1118+480 as a function of the mass transfer rate. One can see that if the donor star has a strong surface magnetic field of $10^{4} \mathrm{G}$ and the mass transfer rate is $\sim 7 \times 10^{-9} M_{\odot} \mathrm{yr}^{-1}$, magnetic braking can indeed reproduce the observed period change. According to Eq. (9) of King et al. (1996), the mass transfer rate driven by magnetic braking can be written as

$-\dot{M}_{\mathrm{d}}=2 \times 10^{-9}\left(\frac{M_{\mathrm{bh}}}{M_{\odot}}\right)^{-2 / 3}\left(\frac{M_{\mathrm{d}}}{M_{\odot}}\right)^{7 / 3}\left(\frac{P}{3 \mathrm{~h}}\right)^{5 / 3} M_{\odot} \mathrm{yr}^{-1}$

For XTE J1118+480, the mass transfer rate is estimated to be $1.6 \times 10^{-11} M_{\odot} \mathrm{yr}^{-1}$. Meanwhile, Wu et al. (2010) found that $3-200 \mathrm{keV}$ peak luminosity of XTE J1118+480 is only about $0.001 L_{\text {Edd }}$, which implied a low mass transfer rate of

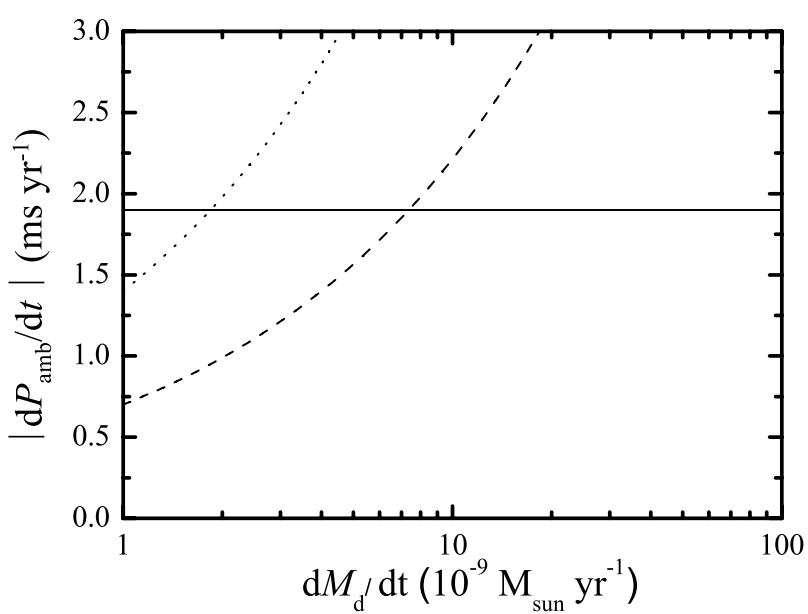

Fig. 1. Orbital-period change rate for XTE J1118+480 predicted by anomalous magnetic braking as a function of the mass transfer rate. The dashed and dotted curves correspond to the surface magnetic fields of $10^{4} \mathrm{G}$ and $2 \times 10^{4} \mathrm{G}$, respectively. The horizontal solid line denotes the observed value of $\mathrm{J} 1118+480$.

$\sim 10^{-10} M_{\odot} \mathrm{yr}^{-1}$. In addition, the standard magnetic braking is thought to terminate in stars with a mass of $\lesssim 0.3 M_{\odot}$, in which the donor stars become completely convective (Rappaport et al. 1983; Spruit \& Ritter 1983). Therefore, some uncertainties still remain about whether the rapid orbital decay of XTE J1118+480 originates from magnetic braking.

\subsection{Circumbinary disk}

Muno \& Mauerhan (2006) have detected the blackbody spectrum of compact BHXBs A 0620-00 and XTE J1118+480, and found that the inferred areas of mid-infrared 4.5-8 $\mu \mathrm{m}$ excess emission are about two times larger than the binary orbital separations. Recently, a detection with the Wide-field Infrared Survey Explorer identified that XTE J1118+480 and A0620-00 are candidate circumbinary disk systems (Wang \& Wang 2014). These two works strongly support the existence of circumbinary disks around compact BHXBs. For the origin of circumbinary disks, the material outflow during the mass transfer may be an alternative route (Dubus et al. 2004) ${ }^{1}$. If a fraction $\delta$ of the transferred mass feeds into the circumbinary disk at its inner radius $r_{i}$, tidal torque exerted on the circumbinary disk can extract the orbital angular momentum from the BHXB. The angular momentum loss rate can be written as (Spruit \& Taam 2001; Taam \& Spruit 2001)

$\dot{J}_{\mathrm{CB}}=\gamma\left(\frac{2 \pi a^{2}}{P}\right) \delta \dot{M}_{\mathrm{d}}\left(\frac{t}{t_{\mathrm{vi}}}\right)^{1 / 2}$,

where $\gamma=\sqrt{r_{\mathrm{i}} / a}, t$ is the mass transfer timescale, and $t_{\mathrm{vi}}$ is the viscous timescale at the inner edge of the disk, $t_{\mathrm{vi}}=$ $2 \gamma^{3} P /\left(3 \pi \alpha \beta^{2}\right)$, where $\alpha$ and $\beta$ are the viscosity parameter and the dimensionless parameter describing the scale height at $r_{\mathrm{i}}$, respectively (Shakura \& Sunyaev 1973). Adopting the typical parameters $\gamma=1.3, \alpha_{\mathrm{SS}}=0.01, \beta=0.03$ (Chen et al. 2006), the orbital period derivative caused by the circumbinary disk is

1 Circumbinary disks were also suggested to be the remnant of fallback disks formed in the supernovae (Wang et al. 2006; Cordes \& Shannon 2008). 
Table 1. Observed parameters of XTE J1118+480 and A0620-00.

\begin{tabular}{llllll}
\hline \hline Sources & $\begin{array}{l}M_{\mathrm{bh}} \\
\left(M_{\odot}\right)\end{array}$ & $\begin{array}{l}M_{\mathrm{d}} \\
\left(M_{\odot}\right)\end{array}$ & $\begin{array}{l}R_{\mathrm{d}} \\
\left(R_{\odot}\right)\end{array}$ & $\begin{array}{l}P_{\text {orb }} \\
(\mathrm{d})\end{array}$ & $\begin{array}{l}\dot{P}_{\text {orb }} \\
\left(\mathrm{ms} \mathrm{yr}^{-1}\right)\end{array}$ \\
\hline J1118+480 & $7.46_{-0.69}^{+0.34}$ & $0.18 \pm 0.06$ & $0.34 \pm 0.05$ & 0.169934 & $-1.90 \pm 0.57$ \\
References & 1 & 1 & 1 & $1,2,3$ & 1 \\
\hline A0620-00 & $6.61_{-0.17}^{+0.23}$ & $0.40 \pm 0.01$ & $0.67 \pm 0.02$ & 0.3230142 & $-0.60 \pm 0.08$ \\
References & 1 & 1 & 1,4 & 1,5 & 1 \\
\hline
\end{tabular}

References. (1) González Hernández et al. (2014); (2) Torres et al. (2004); (3) González Hernández et al. (2012); (4) González Hernández (2011); (5) McClintock \& Remillard (1986).

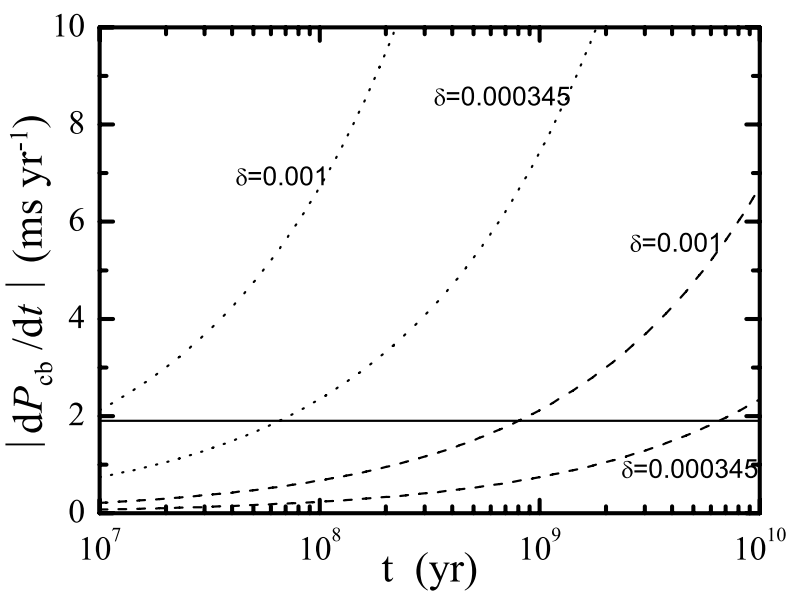

Fig. 2. Orbital-period change rate for XTE J1118+480 caused by a circumbinary disk as a function of the mass transfer timescale. The dashed and dotted curves correspond to the mass transfer rates of $10^{-9}$ and $10^{-8} M_{\odot} \mathrm{yr}^{-1}$, respectively. The horizontal solid line denotes the observed value of $\mathrm{J} 1118+480$.

given by

$\dot{P}_{\mathrm{CB}}=-0.34 \frac{\delta \dot{M}_{\mathrm{d},-9} M_{\mathrm{T}}}{M_{\mathrm{bh}} M_{\mathrm{d}}}\left(\frac{t}{142 \mathrm{yr}}\right)^{0.5}\left(\frac{P}{1 \mathrm{~d}}\right)^{0.5} \mathrm{~ms} \mathrm{yr}^{-1}$,

where $\dot{M}_{\mathrm{d},-9}$ is the mass transfer rate in unit of $10^{-9} M_{\odot} \mathrm{yr}^{-1}$. In Fig. 2, we plot the changing rate of the orbital period for XTE $\mathrm{J} 1118+480$ predicted by the circumbinary disk model (the mass input fraction in the inner edge of circumbinary disk is $\delta=10^{-3}$ ) as a function of the mass transfer timescale. As shown in this figure, if the mass transfer rate is $\sim 10^{-9} M_{\odot} \mathrm{yr}^{-1}$, the evolution can lead to a change rate of $\dot{P} \approx-2.0 \mathrm{~ms} \mathrm{yr}^{-1}$ at $t=10^{9} \mathrm{yr}$. For a small $\delta=0.000345$ (see also Sect. 3), a long mass transfer timescale is expected.

\section{Evolutionary simulation of XTE J1118+480}

As shown in the previous section, an overly high mass transfer rate of $\sim 10^{-8} M_{\odot} \mathrm{yr}^{-1}$ is required to account for the rapid orbital decay in XTE J1118+480 and A0620-00 if magnetic braking works, while the circumbinary disk can address the period derivative observed within a reasonable range of the input parameters. Therefore, based on the circumbinary disk model we used an updated version of the stellar evolution code developed by Eggleton $(1971,1972,1973)$ to simulate the evolution of XTE $\mathrm{J} 1118+480$. For the donor star, we adopt an initial solar chemical composition $(X=0.7, Y=0.28$, and $Z=0.02)$ and a ratio of the mixing length to the pressure scale height of 2.0. In our calculations, we considered four mechanisms that are losing orbital angular momentum, including gravitational radiation, magnetic braking, mass loss, and the effect of circumbinary disk. Therefore, the loss rate in the orbital angular momentum of BHXB can be written as

$\dot{J}_{\mathrm{orb}}=\dot{J}_{\mathrm{GR}}+\dot{J}_{\mathrm{MB}}+\dot{J}_{\mathrm{ML}}+\dot{J}_{\mathrm{CB}}$,

where four terms on the right-hand side denote angular momentum loss rate by gravitational radiation, magnetic braking, mass loss, and the circumbinary disk. If the mass transfer rate is higher than the Eddington accretion rate of the $\mathrm{BH}$, we assume that the redundant transferred matter is ejected in the vicinity of the $\mathrm{BH}$, forming isotropic winds and carrying away the specific orbital angular momentum of the $\mathrm{BH}$. When the donor star mass is in the range of $0.3-1.5 M_{\odot}$, we adopt an empirical angular momentum loss prescription for magnetic braking proposed by Sills et al. (2000).

To fit the observed parameters ( mass and radius of the donor star and orbital period) of XTE J1118+480, we calculated the detailed evolutionary sequences of a BHXB with an initial orbital period of $0.9 \mathrm{~d}$, which consist of a $5.0 M_{\odot} \mathrm{BH}$ and a 3.0 $M_{\odot}$ donor star. The calculation shows that if the fraction of the transferred mass feeds into the circumbinary disk $\delta$ is greater than 0.00035 , the mass transfer becomes dynamically unstable. If we take $\delta=0.000345$, the calculated evolution of the BHXB is in agreement with the observations. For the other result $\delta \leq 0.00034$, the efficiency of circumbinary disk the extracting angular momentum is relatively low and the final orbital period of the binary is obviously higher than the observed value. The detailed results are summarized in Table 2 . One can see that a high $\delta$ result in a high angular momentum loss rate, a rapid mass transfer, and a compact orbit.

In Figs. 3 and 4, we show the detailed evolutionary sequences of a BHXB with an initial orbital period of $0.9 \mathrm{~d}$, which consist of a $5.0 M_{\odot} \mathrm{BH}$ and a $3.0 M_{\odot}$ donor star. In our calculations, we assume that a relatively small fraction $(\delta=0.000345)$ of the transferred material forms a circumbinary disk. At the age of $t \simeq 157.2 \mathrm{Myr}$, rapid mass transfer initiates when the donor star fills its Roche lobe. The H-rich material is transferred onto the $\mathrm{BH}$ at a rate of $\sim 10^{-8} M_{\odot} \mathrm{yr}^{-1}$, which is lower than the Eddington accretion rate of the $\mathrm{BH}$. Because the material is transferred from the less massive donor star to the more massive $\mathrm{BH}$, the orbital period increases to $1.4 \mathrm{~d}$ after $\sim 70 \mathrm{Myr}$ mass transfer. Along with the mass transfer, the tidal torque exerted by the circumbinary disk gradually enhances. When $\dot{P}_{\mathrm{CB}}=\dot{P}_{\mathrm{MT}}$, the orbital period of BHXB begins to decrease and the mass transfer enters a "plateau" phase of $\sim 400 \mathrm{Myr}$ at a rate $\sim 10^{-9} M_{\odot} \mathrm{yr}^{-1}$. Once the donor star ascends the giant branch, the mass transfer rate sharply increases to $3 \times 10^{-8} M_{\odot} \mathrm{yr}^{-1}$, and the orbital period rapidly decreases. At the age of $t \simeq 812.5 \mathrm{Myr}$, the orbital period decreases to $0.17 \mathrm{~d}$, and the $\mathrm{BH}$ and donor masses become $7.81 M_{\odot}$ and $0.185 M_{\odot}$, respectively. Losing most material, the radius of the donor star decreases to be 

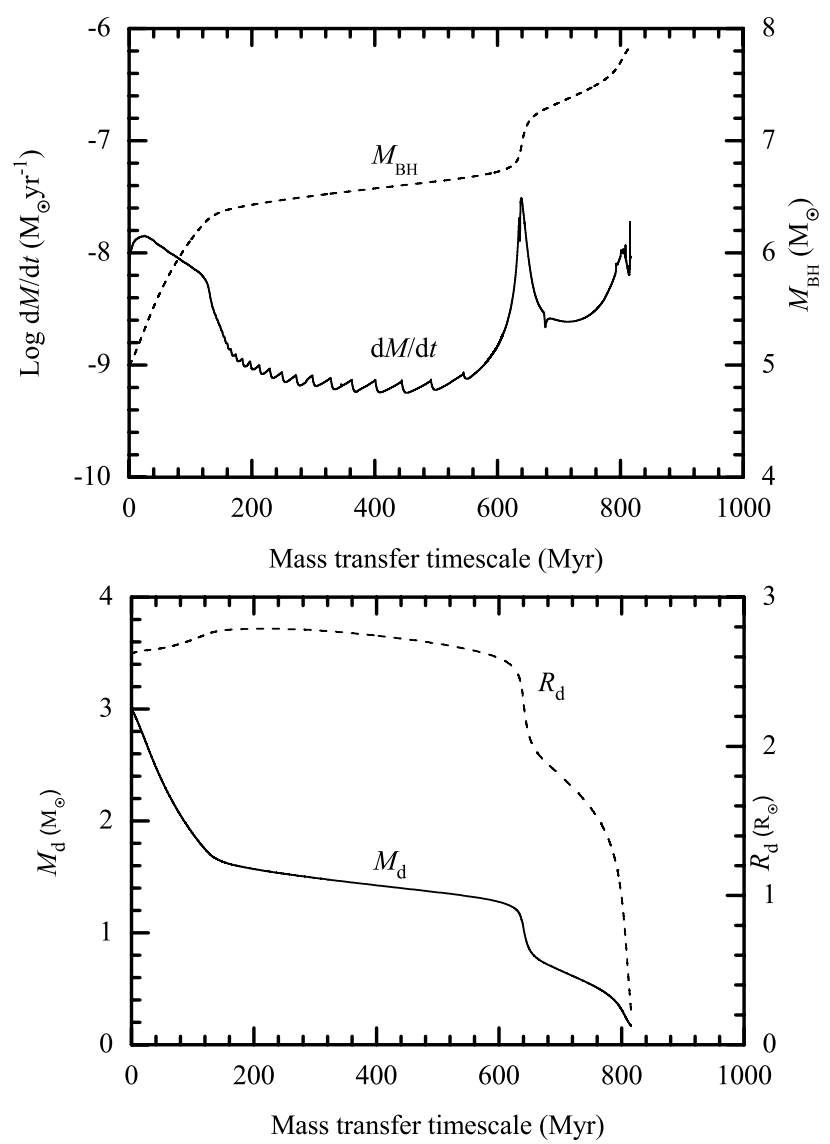

Fig. 3. Evolutionary tracks of BHXB with an initial orbital period of $0.9 \mathrm{~d}$, which consists of a donor star of $M_{\mathrm{d}}=3.0 M_{\odot}$ and a black hole of $M_{\mathrm{bh}}=5.0 M_{\odot}$. The solid and dashed curves represent the evolution of the mass transfer rate, and the $\mathrm{BH}$ mass, in the top panel, and the donor star mass and the donor star radius in the bottom panel, respectively.

$0.336 R_{\odot}$. The tidal torque caused by the circumbinary disk takes away the orbital angular momentum at a rate $-2.76 \mathrm{~ms} \mathrm{yr}^{-1}$, which is in general agreement with the observation. Our simulated results indicate that the circumbinary disk provide an alternative evolutionary channel to form compact BHXBs.

Recently, Fragos et al. (2009) have constrained the progenitor properties of XTE J1118+480, and concluded that the donor star mass is $1.0-1.6 M_{\odot}$, the $\mathrm{BH}$ mass is $6-10 M_{\odot}$, and the orbital period is $0.5-0.8 \mathrm{~d}$ at the beginning of Roche lobe overflow. Therefore, we also calculated the evolutionary sequences a BHXB with a $6.0 M_{\odot} \mathrm{BH}$ and a $1.5 M_{\odot}$ donor star, and an initial orbital period of $0.84 \mathrm{~d}$. Similar to the donor star of $3.0 M_{\odot}$, we also present the simulated result under different input parameters $\delta$ in Table 3 . One can see that the calculated results, when $\delta=0.00018$, can fit the observations. Generally, the mass transfer rates are lower for lower donor masses, at a rate of $\sim 10^{-10}-10^{-9} M_{\odot} \mathrm{yr}^{-1}$. In the final evolutionary stage, the mass transfer rate reached a peak of $\sim 10^{-8}-10^{-7} M_{\odot} \mathrm{yr}^{-1}$. At the age of $t \simeq 2763 \mathrm{Myr}$, the system evolved into a compact BHXB with an orbital period of $0.17 \mathrm{~d}$, which includes a $7.31 M_{\odot} \mathrm{BH}$ and a $0.182 M_{\odot}$ donor star. At this moment, the radius of the donor star is $0.336 R_{\odot}$ and the orbital period derivative is $-2.19 \mathrm{~ms} \mathrm{yr}^{-1}$.

\section{Discussion and summary}

In this work, we have investigated the origin of rapid orbital decay in two compact BHXBs. Our analysis shows that even if

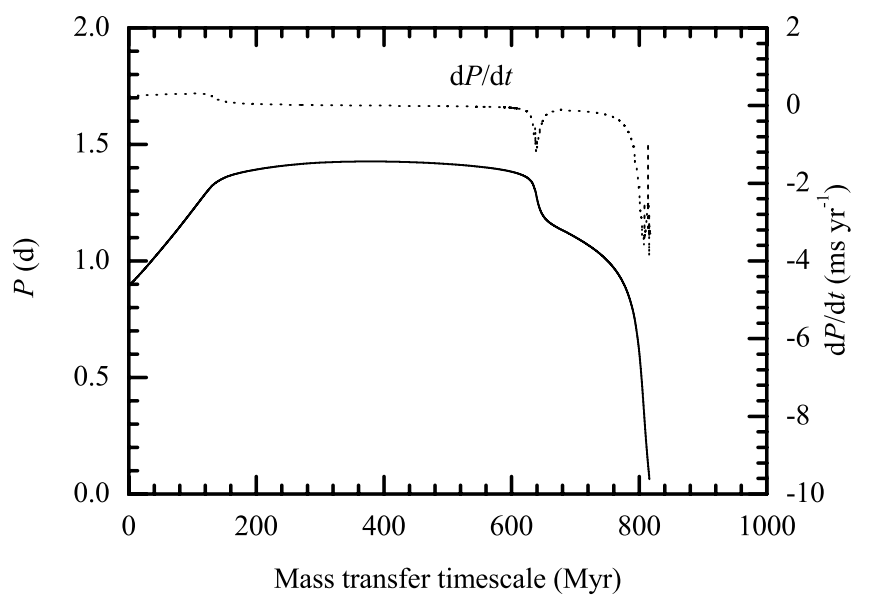

Fig. 4. Evolutionary tracks of BHXB with an initial orbital period of $0.9 \mathrm{~d}$, which consist of a donor star of $M_{\mathrm{d}}=3.0 M_{\odot}$ and a black hole of $M_{\mathrm{bh}}=5.0 M_{\odot}$. The solid and dotted curves represent the evolution of the orbital period and orbital period derivative, respectively.
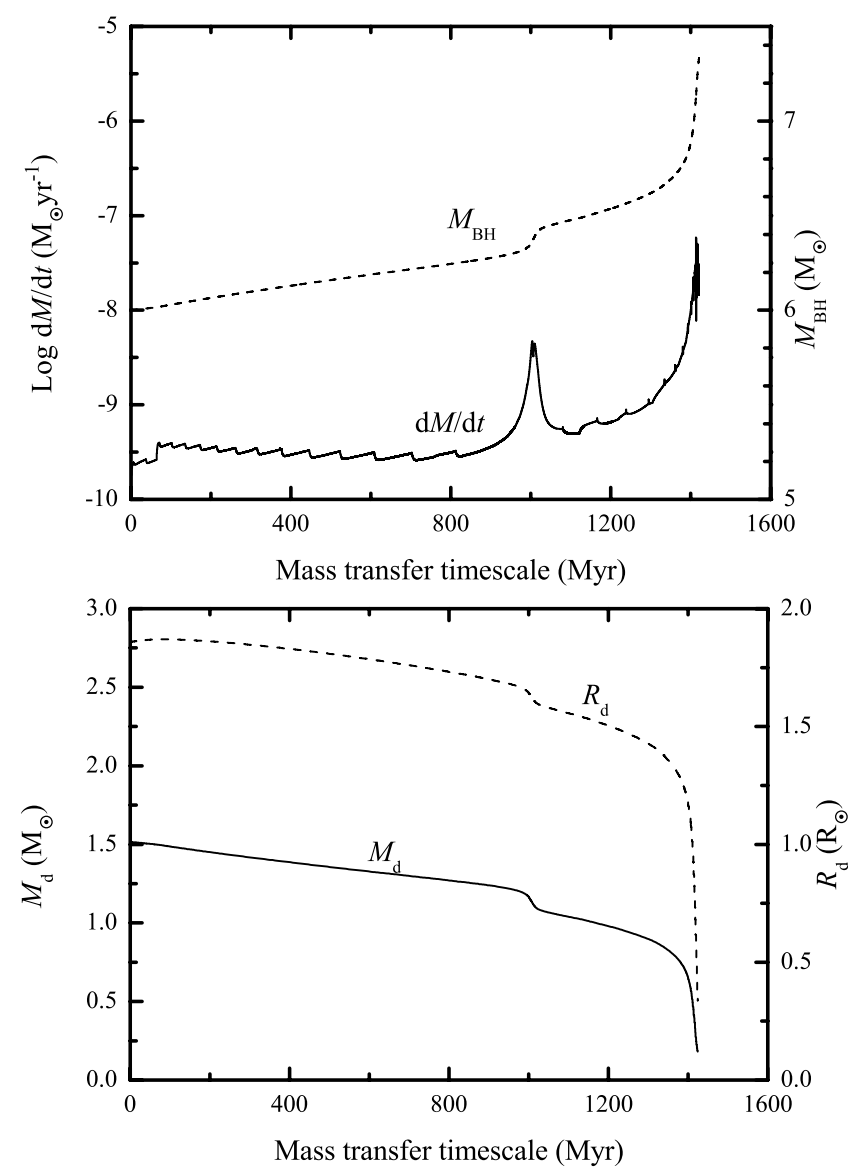

Fig. 5. Evolutionary tracks of BHXB with an initial orbital period of $0.84 \mathrm{~d}$, which consists of a donor star of $M_{\mathrm{d}}=1.5 M_{\odot}$ and a black hole of $M_{\mathrm{bh}}=6.0 M_{\odot}$. The solid and dashed curves represent the evolution of the mass transfer rate and $\mathrm{BH}$ mass in the top panel, and the donor star mass and donor star radius in the bottom panel, respectively.

the donor stars of BHXBs are Ap/Bp stars with a strong magnetic fields of 10-20 kG, anomalous magnetic braking scenario still needs to solve two key problems: the mass transfer rate and the cutoff of magnetic braking below $0.3 M_{\odot}$. Some observations indicate that circumbinary disks around compact BHXBs XTE J1118+480 and A0620-00 may exist. It is a coincidence 
Table 2. Calculated result of BHXBs with a donor star of $3 M_{\odot}$ under different input parameters $\delta$.

\begin{tabular}{cccccc}
\hline \hline Item & $\delta$ & $\begin{array}{c}M_{\text {bh }} \\
\left(M_{\odot}\right)\end{array}$ & $\begin{array}{c}M_{\mathrm{d}} \\
\left(M_{\odot}\right)\end{array}$ & $\begin{array}{c}P_{\text {orb }} \\
(\mathrm{d})\end{array}$ & $\begin{array}{c}\dot{P}_{\text {orb }} \\
\left(\mathrm{ms} \mathrm{yr}^{-1}\right)\end{array}$ \\
\hline $1^{*}$ & 0.0002 & 7.70 & 0.29 & 48.97 & 1.63 \\
$2^{*}$ & 0.0003 & 7.77 & 0.23 & 9.58 & 0.22 \\
3 & 0.00034 & 7.82 & 0.18 & 0.54 & -0.12 \\
4 & 0.000345 & 7.82 & 0.18 & 0.17 & -2.76 \\
5 & 0.00035 & 7.82 & 0.18 & 0.15 & -2.30 \\
\hline
\end{tabular}

Notes. ${ }^{(*)}$ When the binary system has already evolved into a detached system, the donor star mass is still greater than $0.18 M_{\odot}$.

Table 3. Calculated result of BHXBs with a donor star of $1.5 M_{\odot}$ under different input parameters $\delta$.

\begin{tabular}{cccccc}
\hline \hline Item & $\delta$ & $\begin{array}{c}M_{\mathrm{bh}} \\
\left(M_{\odot}\right)\end{array}$ & $\begin{array}{c}M_{\mathrm{d}} \\
\left(M_{\odot}\right)\end{array}$ & $\begin{array}{c}P_{\text {orb }} \\
(\mathrm{d})\end{array}$ & $\begin{array}{c}\dot{P}_{\text {orb }} \\
\left(\mathrm{ms} \mathrm{yr}^{-1}\right)\end{array}$ \\
\hline $1^{a}$ & 0.0001 & 7.26 & 0.25 & 12.17 & 0.03 \\
$2^{a}$ & 0.00015 & 7.31 & 0.21 & 2.19 & 0.00 \\
3 & 0.00017 & 7.34 & 0.18 & 0.59 & -0.01 \\
4 & 0.00018 & 7.33 & 0.18 & 0.17 & -2.19 \\
$5^{b}$ & 0.0002 & - & - & - & - \\
\hline
\end{tabular}

Notes. ${ }^{(a)}$ When the binary system has already evolved into a detached system, the donor star mass is still greater than $0.18 M_{\odot}{ }^{(b)}$ The mass transfer is dynamically unstable.

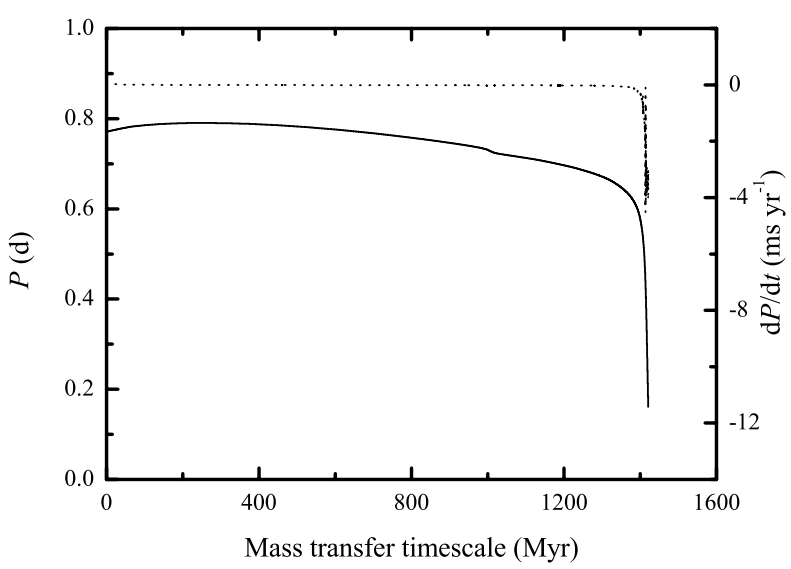

Fig. 6. Evolutionary tracks of BHXB with an initial orbital period of $0.84 \mathrm{~d}$, which consists of a donor star of $M_{\mathrm{d}}=1.5 M_{\odot}$ and a black hole of $M_{\mathrm{bh}}=6.0 M_{\odot}$. The solid and dotted curves represent the evolution of the orbital period and orbital period derivative, respectively.

that rapid orbital decay have been discovered in two compact BHXBs. If the mass transfer timescale is $10^{9} \mathrm{yr}$ and the mass transfer rate is $\sim 10^{-9} M_{\odot} \mathrm{yr}^{-1}$, the period derivative caused by circumbinary disk with $\delta=0.001$ can account for the observation.

Based on the circumbinary disk model, we performed numerical calculations for the evolution of BHIMXB with $3.0 M_{\odot}$ and $1.5 M_{\odot}$ donor star, and an initial orbital period of $0.9 \mathrm{~d}$, and $0.84 \mathrm{~d}$, respectively. Our simulated results, including the black hole mass, donor star mass, donor star radius, orbital period, and orbital period derivative, are approximately in agreement with the observed parameters of XTE J1118+480. It seems that the circumbinary disk can be an alternative scenario to account for the rapid orbital decay observed in some compact BHXBs. However, our simulations show that the mass transfer rate is about $\sim 10^{-8} M_{\odot} \mathrm{yr}^{-1}$ at the current stage of XTE J1118+480, which is obviously two orders of magnitude higher than the inferred value from observed luminosity ( $\mathrm{Wu}$ et al. 2010). Furthermore, there is a significant discrepancy between our simulated mass and the observed mass of the circumbinary disk. If the material of the circumbinary disk does not decrease during the evolution, the mass of the circumbinary disk can be estimated to be $\sim 10^{-9} M_{\odot} \mathrm{yr}^{-1} \times 10^{9} \mathrm{yr} \times$ $0.000345(0.00018)=0.000345(0.00018) M_{\odot}$. This mass are five to six orders of magnitude higher than the inferred mass $\left(\sim 10^{-9} M_{\odot}\right)$ of the circumbinary disk around XTE J1118+480 (Muno \& Mauerhan 2006). Specially, Gallo et al. (2007) suggested that nonthermal synchrotron emission from a jet could account for a significant fraction (or all) of the measured excess mid-IR emission. Therefore, the circumbinary disk mass may be even lower. The circumbinary disk should not be the main mechanism causing the rapid orbital decay observed in some compact black hole X-ray binaries because the mass transfer rate and circumbinary disk mass are obviously far greater than the inferred values from observations (González Hernández et al. 2012).

Acknowledgements. We are grateful to the anonymous referee for helpful comments. This work was partly supported by the National Science Foundation of China (under grant number 11133001, 11173018, and 11333004), and Innovation Scientists and Technicians Troop Construction Projects of Henan Province, China.

\section{References}

Casares, J. 2007, in Black Holes from Stars to Galaxies - Across the Range of Masses, eds. V. Karas \& G. Matt (Cambridge: Cambridge Univ. Press), IAU Symp., 238, 3

Chen, W.-C., \& Li, X.-D. 2006, MNRAS, 373, 305

Chen, W.-C., Li, X.-D., \& Qian, S.-B. 2006, ApJ, 649, 973

Cordes, J. M., \& Shannon, R. M. 2008, ApJ, 682, 1152

Dervişoǧlu, A., Tout, C. A., \& Ibanoǧlu, C. 2010, MNRAS, 406, 1071

Dubus, G., Campbell, R., Kern, B., Taam, R. E., \& Spruit, H. C. 2004, MNRAS, 349, 869

Eggleton, P. P. 1971, MNRAS, 151, 351

Eggleton, P. P. 1972, MNRAS, 156, 361

Eggleton, P. P. 1973, MNRAS, 163, 279

Fragos, T., Willems, B., Kalogera, V., et al. 2009, ApJ, 697, 1057

Gallo, E., Migliari, S., Markoff, S., et al. 2007, ApJ, 670, 600

González Hernández, J. I., Casares, J., Rebolo, R., et al.. 2011, ApJ, 738, 95

González Hernández, J. I., Rebolo, R., \& Casares, J. 2012, ApJ, 744, L25

González Hernández, J. I., Rebolo, R., \& Casares, J. 2014, MNRAS, 438, L21

Haswell, C. A., Hynes, R. I., King, A. R., \& Schenker, K. 2002, MNRAS, 332, 928

Justham, S., Rappaport, S., \& Podsiadlowski, Ph. 2006, MNRAS, 366, 1415

Kawaler, S. D. 1988, ApJ, 333, 236

King, A. R., Kolb, U., \& Burderi, L. 1996, ApJ, 464, L127

Lee, C. -H., Brown, G. E., \& Wijers, R. A. M. J. 2002, ApJ, 575, L996

McClintock, J. E., \& Remillard, R. A. 1986, ApJ, 308, 110

Muno, M. P., \& Mauerhan, J. 2006, ApJ, 648, L135

Podsiadlowski, Ph., Rappaport, S., \& Han, Z. 2003, MNRAS, 341, 385

Rappaport, S., Verbunt, F., \& Joss, P. C. 1983, ApJ, 275, 713

Remillard, R. A., \& McClintock, J. E. 2006, ARA\&A, 44, 49

Ritter, H., \& Kolb, U., 2003, A\&A, 404, 301

Shakura, N. I., \& Sunyaev, R. A. 1973, A\&A, 24, 337

Sills, A., Pinsonneault, M. H., \& Terndrup, D. M. 2000, ApJ, 534, 335

Spruit, H. C., \& Ritter, H. 1983, A\&A, 124, 267

Spruit, H. C., \& Taam, R. E. 2001, ApJ, 548, 900

Taam, R. E., \& Spruit, H. C. 2001, ApJ, 561, 329

Torres, M. A. P., Callanan, P. J., Garcia, M. R., et al. 2004, ApJ, 612, 1026

Verbunt, F., \& Zwaan, C. 1981, A\&A, 100, L7

Wang, X., \& Wang, Z. 2014, ApJ, 788, 184

Wang, Z., Chakrabarty, D., \& Kaplan, D. L. 2006, Nature, 440, 772

Wu, Y. X., Yu, W., Li, T. P., Maccaronae, T. J., \& Li, X. D. 2010, ApJ, 718, 620 